\title{
The Realization of the "New Architecture". The Italian Colonial Experience in Albania During the Years 1925-1943.
}

\author{
Ariola Prifti \\ Center of Albanological Studies \\ Head of Ethnographic funds \\ Sheshi "Nene Tereza", Nr. 3, Tirana, Albania \\ ariola_prifti@yahoo.it
}

Doi:10.5901/ajis.2013.v2n9p648

\begin{abstract}
The Italian colonial architecture is a chapter yet to be completed, although in recent years there has been a keen interest in the topic, which has led to important events. The period of greatest expansion of Italian colonialism is the one between the second half of the twenties, when fascism began the arrangement of the "Terre d'Oltremare" and the important year 1936, when was made the proclamation of the Empire. The Italian colonial developments in Albania was so particular. They were expected economic agreements for the construction of public works, financial assistance to the country, and its entry into the Society of Nations. The present study was conceived with the aim of identifying a specific strand of the Italian colonial, that is what born inside of the Rationalism, and eventually the emergence of colonial and modern architecture.In 1928 were founded the National Bank of Albania, a subsidiary of the Bank of Italy, and the SVEA which guaranteed, plus a huge loan, the use of companies with Italian capital for the construction of the road network and the completion of the new capital Tirana, whose plan was entrusted to architect Armando Brasini. In the Italian colonies have worked among the best urban planners and architects of the time, each with its own training and their militancy in the various movements of the era. Finally, these great masters of architecture studied the ancient local construction systems and materials used, replugging the achievements of modern technology with the great heritage of tradition. To prepare this study researched a wide historic literature and archive resources, which tried to analyze and interpret according to the present arguments.
\end{abstract}

Keywords: The Italian colonial experience, New architecture, Italian colonialism, Modern architecture.

\section{Introduzione}

Le storie dell'Italia e dell'Albania si sono ripetutamente intrecciate nel corso dei secoli. Da questo lungo cammino, segnato da uno scambio intenso di esperienze e di conoscenze, ma anche da fasi di grave e lacerante contrapposizione è nato un rapporto di amicizia solido e profondo, alimentato dalla comune appartenenza alla civiltà del Mediterraneo e dalla condivisione dei valori di accoglienza e di solidarietà che di quella civiltà rappresentano il tratto distintivo. Oggi fanno da testimonianza della presenza italiana in Albania nella prima metà del Novecento, un insieme di documenti archivistici, dove si nota particolarmente il ruolo che l'architettura e la pianificazione del territorio hanno svolto come veicolo di diffusione della cultura italiana nell'Albania di questo periodo. Questo legame di cooperazione si nota ancora nei giorni nostri, tra progettisti e costruttori dei due Paesi nella realizzazione di alcune tra le più significative espressioni del patrimonio artistico albanese contemporaneo.

Si è trattato di un ambito in cui ha dunque trovato riflesso il dialogo costante e proficuo tra i due Paesi. Che continua ancora oggi in forme diversificate, estendendosi dal partenariato commerciale agli scambi socio-culturali.

\section{Gli obiettivi del proggetto}

Il presente studio è nato con l'obiettivo di identificare un preciso filone dell'architettura coloniale italiana nell'Albania degli anni tra 1925-1943, ossia quello che nasce all'interno del Razionalismo, e alla fine l'affermazione di un'architettura coloniale moderna.

Le colonie italiane costituirono nel loro complesso un universo variegato, per la diversità delle caratteristiche geografiche, storiche e culturali. Per questo le politiche urbanistiche ed edilizie del Fascismo nelle colonie furono diverse una dall'altra. 
L'Albania viene considerato un caso a parte, trattandosi di un protettorato, presentava punti di contatto con l'Italia per ragioni storiche e culturali. II divario era inoltre allargato da uno sviluppo storico e da manifestazioni culturali totalmente differenti. Per questo motivo l'organizzazione del territorio, l'urbanistica e l'architettura nelle colonie assunsero forme specifiche, adattandosi volta per volta al contesto, perché i problemi da risolvere erano di varia natura.

Allo scopo di comparare le diverse esperienze, si è voluto tracciare un quadro generale delle maggiori realizzazioni coloniali del Fascismo nel campo della pianificazione territoriale e dell'urbanistica, all'interno del quale risultasse più agevole collocare le opere degli architetti coloniali studiati.

L'architettura coloniale italiana è un capitolo ancora da completare, nonostante negli ultimi anni si sia registrato un vivo interesse per l'argomento, che ha portato a eventi importanti. II periodo di massima espansione del colonialismo italiano è quello compreso tra la seconda metà degli anni Venti, quando il Fascismo avviò la sistemazione delle «Terre d'Oltremare» ${ }^{1}$, e il 1936, l'anno della proclamazione dell'Impero. Erano previsti degli accordi economici per la realizzazione di opere pubbliche, un'assistenza finanziaria al paese, e il suo ingresso nella Società delle Nazioni.

\section{Gli intreci tra la storia e gli sviluppi urbanistici del paese.}

La vicenda coloniale italiana in Albania ebbe sviluppi del tutto particolari. La storia recente del paese risaliva alla spartizione dell'area balcanica che le grandi potenze europee iniziarono con il Congresso di Berlino del 1878. La «questione albanese» emerse all'inizio del Novecento, anche se durante i lavori del Congresso berlinese era nato un «Comitato centrale per la difesa dei diritti della nazione Albanese», sostenuto dalla Turchia, che elaborò il progetto di uno stato autonomo, tentando di sottrarsi alle influenza dell'Impero ottomano e degli stati occidentali.

Nei primi anni del secolo accaddero molti eventi che cambiarono il quadro dei rapporti internazionali: la presa del potere dei «giovani turchi», le annessioni della Bosnia-Erzegovina all'Austria e di Creta alla Grecia, la proclamazione dell'indipendenza in Bulgaria, le mire dell'Italia sull'Egeo e sulla Libia. In questa complessa situazione agirono i patrioti albanesi. Nel 1912 il Comitato, riunitosi a Valona, proclamò l'indipendenza del paese, che sarà sancita nel 1913 dal Congresso di Bucarest e, nell'anno successivo, da quello di Londra.

Nel 1914 a Firenze una «Commissione per la definizione dei confini», su mandato della Società delle Nazioni, stabili la divisione dei territori albanesi in mano alla Turchia tra la Grecia, la Serbia e il Montenegro. Sul nuovo trono dell'Albania fu posto Guglielmo di Wied, che fece una politica filoaustriaca, entrata in crisi allo scoppio della prima guerra mondiale.

All'inizio del conflitto l'Albania fu invasa da Greci, Montenegrini e Serbi, mentre il 28 dicembre 1916 le truppe italiane sbarcarono a Valona e nel 1915 liberarono dai Greci gran parte dell'Albania meridionale, mentre nel 1916 fermarono l'avanzata austro-tedesca alla Voiussa. Nel 1917 l'Italia, con il proclama di Argirocastro, si impegnò a garantire l'indipendenza dell'Albania, a ritirarsi dai territori occupati e a facilitare la formazione di un governo autonomo. Erano previsti anche accordi economici per la realizzazione di opere pubbliche, un'assistenza finanziaria al paese, e il suo ingresso nella Società delle Nazioni.

Con l'affermazione del Regime fascista, Ahmed Zogu, che nel 1924 aveva consolidato il potere grazie all'appoggio iugoslavo, adottò nel 1927 una politica favorevole all'Italia, che da tempo dava al paese aiuti economici e finanziari per valorizzarne le risorse. Nel 1928 vennero fondate la Banca Nazionale d'Albania, una filiazione della Banca d'Italia, e la S.V.E.A. (Società Sviluppo Economico Albania) che garantiva, oltre a un ingente prestito, l'impiego di imprese con capitale italiano per la realizzazione della rete stradale e il completamento della nuova capitale Tirana, il cui piano regolatore venne affidato ad Armando Brasini².

Questo particolare rapporto politico venne consolidato con il «Patto d'amicizia», firmato tra i due paesi nel 1926 a Tirana, che fece dell'Albania a tutti gli effetti un protettorato italiano. Intanto Zogu, nominata un'Assemblea costituente, il $1^{\circ}$ settembre 1928 si fece eleggere re.

Tra le prime opere pubbliche a essere realizzate vi furono i porti di Valona e di Durazzo

\footnotetext{
${ }^{1}$ Per il piano regolatore di Rodi cfr. P. Sica, Storia dell'Urbanistica. III, 2. II Novecento, Roma-Bari, Editori Laterza, 1978, p. 508; F.I. Apollonio, Architettura e città nel Dodecaneso, in Architettura italiana d'oltremare 1870-1940, cit., pp. 316-319; S. Martinoli, E. Perotti, Architettura coloniale italiana nel Dodecaneso. 1912-1943, cit., pp. 457-464.

${ }^{2}$ G. Gresleri, La via dell'est: da Tirana a Lubiana, in Architettura italiana d'oltremare 1870-1940, a c. di G. Gresleri, P.G. Massaretti, S. Zagnoni, Venezia, Marsilio Editori, 1993, pp. 323-331. Cfr. L. Billeri, Bosio e i piani urbanistici per le città dell'Albania, in Gherardo Bosio. Architetto fiorentino. 1903-1941, cit., pp. 75-86.
} 
progettati dall'ingegner Luigi Luiggi, autore anche di quelli di Massaua e di Tripoli. Venne poi redatto un piano per la costruzione di nuove strade lungo le direttrici Tirana-Durazzo, Scutari-Elbasan, Durazzo-Valona, con un centinaio di ponti. Presso il Ministero dei Lavori Pubblici venne istituito un apposito Ufficio Tecnico con l'incarico di preparare i piani regolatori dei principali centri urbani albanesi, mentre a Florestano di Fausto e a Vittorio Morpurgo furono affidati incarichi per la progettazione degli edifici ministeriali di Tirana e di Durazzo e di altre architetture pubbliche e private.

Nel 1925 Brasini studiò il piano regolatore di Tirana, il cui elemento principale era un grande viale, orientato secondo la direzione nord-sud, che separava la città esistente dalla sua periferia; su di esso erano allineate architetture monumentali che ne facevano un centro direzionale del tutto autonomo e avulso dal contesto. La sua funzione era quella di una cerniera fra il nucleo antico e la città moderna, per la quale non venivano fornite precise indicazioni. Nel 1939 anche Gherardo Bosio, nel disegnare il piano regolatore definitivo, conserverà l'asse monumentale previsto da Brasini, qualificandolo con un'edilizia di stampo classicista adeguata al ruolo della capitale.

Tra il 1927 e il 1931 l'ambizioso programma di opere pubbliche per l'Albania provocò una crisi tra la S.V.E.A., il governo italiano e quello albanese, che nel 1929 aveva contratto un debito enorme. II Tesoro italiano fu quindi costretto ad anticipare grandi somme come prestito per i lavori pubblici, ma il credito servì per attrarre ulteriormente l'Albania nell'orbita politica nazionale.

Sulla base agli accordi economici presi tra i due paesi, vennero decisi altri importanti lavori. Alla fine del 1929, dopo un sopralluogo di Luiggi ${ }^{3}$ a Durazzo, il Ministero dei Lavori Pubblici fece approntare un programma operativo che dette l'avvio all'attività edilizia. Tra le realizzazioni vi furono la ferrovia Durazzo-Tirana, nuovi ponti, sei edifici ministeriali, il rifacimento della Villa Reale, su progetto di Di Fausto, e il Grand Hotel di Tirana (Hotel Dajti). II completamento della rete stradale migliorò i trasporti, favorendo l'esportazione dei prodotti locali e l'importazione di materie prime e manodopera dall'talia. Tra l'economia nazionale e quella albanese si venne a creare una dipendenza tale che la Banca d'Italia pensò di istituire una moneta unica, ma il timore di una svalutazione della moneta albanese, fece rinviare il proposito. Intanto venne ventilata l'ipotesi di regolare la questione allontanando re Zogu.

Nel 1938 fu inaugurata a Tirana la nuova sede della Banca d'Italia progettata da Vittorio

Morpurgo. L'A.G.I.P, attiva fin dagli anni Trenta nei comprensori di Devoli e Valona, consolidò la sua presenza con l'Azienda Italiana Petroli Albanesi, la cui sede centrale fu disegnata da Angiolo Mazzoni che nel 1939 progettò anche gli uffici dell' A.N.I.C.

Nel 1939, quando Zogu, su pressione francese e inglese, tentò di svincolarsi dalla tutela italiana, Mussolini dichiarò che l'unica soluzione possibile per l'Albania era la sua annessione all'Italia con mezzi militari; perciò dette incarico al ministro degli Esteri Galeazzo Ciano di avviare trattative con le grandi potenze per ottenerne l'assenso, mentre Zogu cercava accordi diplomatici. L'occupazione militare avvenne dal 7 aprile 1939 con lo sbarco a Durazzo e si concluse alla fine del mese quando Vittorio Emanuele III assunse il titolo di «Re d'Italia e d'Albania».

In questa situazione di instabilità politica fu deciso l'aumento della spesa per i lavori pubblici albanesi, in particolare per il completamento del centro di Tirana, dove erano in fase di ultimazione i progetti di Di Fausto. Subito dopo venne costruito un villaggio4 "Pater", dal nome dell'impresa milanese produttrice di prefabbricati, con 500 alloggi per famiglie italiane, mentre l'E.T.A.L. (Ente Turistico e Alberghiero della Libia), realizzava vari Hotel a Durazzo, Tirana e in altri centri.

Si avviò anche un programma di valorizzazione agricola, nella quale i poderi risultanti dalla bonifica integrale venivano organizzati, utilizzando come modelli i villaggi libici ed etiopici. Fu scelta un'area pianeggiante con terreni fertili in prossimità di Tirana e il comprensorio venne diviso in due zone, dove dovevano insediarsi 900 famiglie italiane e 2.000 albanesi. La gestione dei terreni era però di tipo cooperativistico, basato su un sistema di affitto che in breve tempo avrebbe permesso ai coloni di diventare proprietari dei fondi. Le abitazioni, disposte a gruppi, erano situate al centro dei poderi e formavano 6 villaggi, con una popolazione di 8.000 unità distribuita su circa 36.000 ettari coltivabili.

Nell'estate del 1939 venne inviato a Tirana Gherardo Bosio con l'incarico di organizzare un Ufficio Centrale per l'Edilizia e l'Urbanistica che portasse a compimento il piano regolatore. II problema più urgente che si presentava era l'individuazione e l'organizzazione di nuove aree dove far sorgere i nuovi quartieri residenziali destinati alla popolazione italiana, che abitava all'interno della città antica. Bosio assunse la direzione del nuovo ufficio, iniziò la revisione del

\footnotetext{
${ }^{3}$ Luigi Luiggi (Genova, 1856 - Roma 1931) era un esperto nella progettazione di bacini portuali e di condutture idrauliche; conosceva inoltre le teorie e le tecniche urbanistiche più avanzate, e i più importanti esempi di citta coloniali francesi e inglesi.

${ }^{4}$ Le città di fondazione degli anni Trenta dall'Italia all'Oltremare, a c. di R. Besana, C.F. Carli, L. Devoti, L. Prisco, Milano, T.C.I., 2002, pp. 250-253.
} 
vecchio piano e nel 1940 completò la stesura del nuovo regolamento edilizio. Nel progetto erano previste tipologie edilizie uniformi adeguate all'impianto razionale del piano, ma erano contenute anche indicazioni precise sulle altezze, sul verde, sull'arretramento dei blocchi rispetto al filo stradale, sull'uso dei portici, precisando anche i materiali e i colori da usarsi nelle facciate. Di particolare interesse, all'interno del piano regolatore, era la città-giardino a sud di Viale Mussolini, con al centro il quartiere I.N.C.I.S, progettato da Piero Bartolini, un collaboratore di Bosio che, chiamato a Tirana, organizzò I'Ufficio del Piano Regolatore ${ }^{5}$ e seguì la realizzazione dell'intero lotto dell'I.N.C.I.S., composto da grandi blocchi a due livelli con una corte aperta e da alloggi di tre e quattro piani, per un totale di 16 edifici ${ }^{6}$ rimasti interrotti allo scoppio della guerra.

La maggior parte degli architetti europei moderni, da Le Corbusier a Mies Van Der Rohe, furono impressionati da queste costruzioni, nelle quali vedevano realizzati, in forma naturale e senza mediazioni intellettualistiche, i principali obiettivi perseguiti dalla nuova architettura: la funzionalità, l'assenza della decorazione, l'economia, l'uso razionale dei materiali.

Fig. 1. Hotel Dajti, Tirana 1939
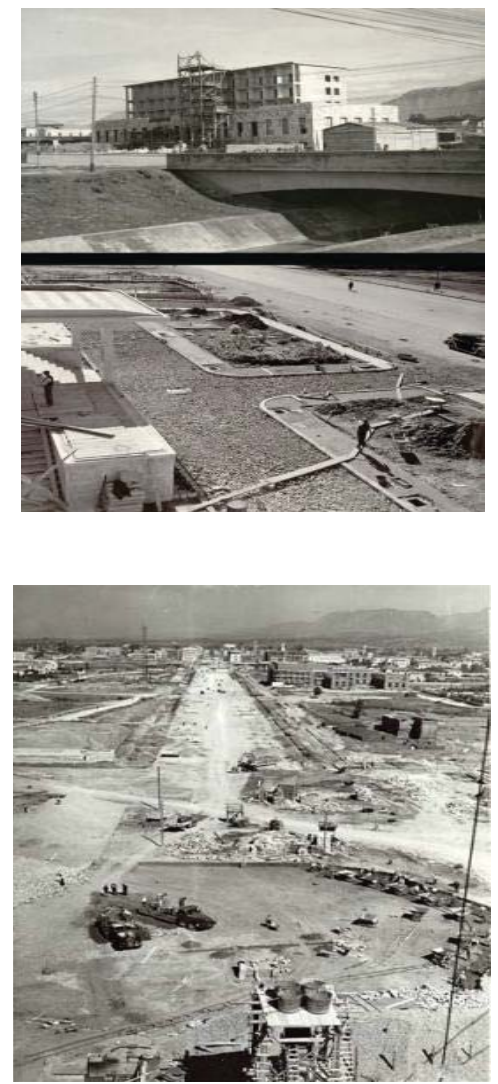

Fig. 2. L'asse principale di Tirana fotografato durante la costruzione del Palazzo di Fascio, oggi Corpo Centrale dell'Università Politecnico di Tirana Luglio 1939

\footnotetext{
${ }^{5}$ Maria Adriana Giusti. Albania, Architettura e Citta' 1925-1943. (2006) Finito di stampare nella tipografia Bandecchi \& Vivaldi, Pontedera per conto di artout - Maschietto Editore, Firenze, Dicembre 2006.

${ }^{6}$ K. Miho, I. Sukaj, P. Thomo, V. Bushati. Historia e arkitektures ne Shqiperi (1912-1944). (1988) Shtepia Botuese e Librit Universitar.Tirane, 1988, 40.
} 


\section{Gli influssi del Razionalismo sull'architettura coloniale italiana}

Lo scopo di questa indagine si fonda sull'individuazione, all'interno dell'esperienza progettuale nelle colonie italiane ${ }^{7}$, di una «nuova architettura», intendendo con il termine quella più avanzata dal punto di vista linguistico e ben distinta dall'architettura ufficiale 0 da quella che attingeva le proprie ragioni teoriche e la propria poetica da altri movimenti dell'epoca. La sua origine è da rintracciare verso la fine degli anni Venti nelle ricerche maturate nel Razionalismo ${ }^{8}$ italiano, dove si formarono i giovani architetti italiani che ebbero l'occasione di lavorare nelle colonie. Le loro opere si differenziarono in misura notevole da quelle degli altri architetti.

Nella costruzione dell'apparato "ideologico" di questa «nuova architettura» coloniale avranno un peso rilevante alcune "matrici" culturali presenti nel dibattito architettonico dell'epoca, svoltosi nella madrepatria negli anni Trenta e oltre, di cui i razionalisti furono tra i più ferventi animatori.

Tra queste, le più importanti ai fini del nostro discorso sono tre, mentre in realtà sono strettamente connesse e interdipendenti, rimandando a una sostanziale unità. La prima matrice è l'architettura mediterranea, intesa come un ambito di appartenenza più vasto di quello nazionale, al quale ricondurre l'architettura moderna, per una coincidenza di principi e di fatti formali. La seconda, che costituisce una derivazione della prima, è la rivalutazione dell'architettura "senza architetti" dell'Italia del sud, delle isole greche e delle coste nordafricane, nella quale si riteneva fossero contenute le fonti della razionalità costruttiva. Questi due temi nacquero e si affermarono nella cerchia del Razionalismo, anche se in seguito altri gruppi dell'epoca si approprieranno del concetto della mediterraneità. La terza matrice è la ricerca dell'identità dell'architettura italiana moderna: un obiettivo che nelle colonie si poneva in maniera più evidente, in quanto si trattava di costruire in un contesto totalmente estraneo a quello del paese di origine. Ma questa finalità fu perseguita anche da altre correnti, soprattutto da quella accademica, come resistenza alla diffusione internazionale della nuova architettura. Nei razionalisti la ricerca delle radici nazionali scaturisce dal rapporto dialettico tra l'avanguardia e la tradizione e sarà impostata, anche per un calcolo politico, sull'eredità dell'architettura romana; di essa però i giovani architetti attivi nelle colonie non guarderanno gli esempi aulici e monumentali, ma la domus, considerata come l'archetipo dell'abitazione del bacino mediterraneo. Queste tre griglie di interpretazione rappresentano anche altrettante coordinate atte a orientarsi nella ricostruzione dell'arduo percorso a ritroso degli architetti coloniali razionalisti. Partiti dal movimento europeo, essi vollero realizzare un'architettura moderna ma anche italiana, e perciò andarono alla ricerca dei suoi caratteri permanenti, che ritrovarono nell'edilizia spontanea dell'area mediterranea, dove lavorarono. Qui essi scoprirono il valore degli antichi centri urbani e dell'abitazione indigena, della quale studieranno la morfologia, l'uso razionale dei materiali, il rapporto con l'ambiente naturale e le soluzioni per il controllo climatico. Abbandonando infine gli estremismi dell'avanguardia, si ricollegarono alla tradizione, di cui accettarono gli insegnamenti, rivisitati però alla luce dei principi del Movimento Moderno, realizzando un'architettura contestuale ante litteram.

\section{I risultati e le discussioni}

Gli interessi italiani verso l'Albania ${ }^{9}$ all'inizio del '900 traevano origine dalla necessità di esercitare un controllo strategico sull'Adriatico attraverso il possesso delle due sponde del Canale d'Otranto e dall'opportunità di servirsi della terra albanese per una penetrazione economico-finanziaria nell'area balcanica. A questi chiari obiettivi, tuttavia, non fece mai riscontro, un indirizzo politico altrettanto determinato: difatti, se i governi liberali operarono sempre con incertezza, neppure il governo fascista seppe distaccarsi da questo approccio. Mussolini condusse nei confronti dell'Albania una politica ambigua che mirava ad esercitare un controllo indiretto attraverso una sorta di protettorato, ma non scartava neanche la possibilità di un'occupazione militare. Anche i piani elaborati dalle Forze Armate italiane dal 1927 in poi, risentirono di questo duplice orientamento, il quale finì per riflettersi negativamente sull'ultimo atto della politica fascista verso l'Albania di Zog, tanto che l'azione delle truppe italiane nell'aprile del 1939 non si tradusse in un disastro solo per l'assoluta inconsistenza dell'avversario. La lunga gestazione dei progetti militari per l'Operazione "Oltre Mare Tirana" e i

\footnotetext{
7 Vittorio Santoianni, II Razionalismo nelle colonie italiane 1928-1943. La «nuova architettura» delle Terre d'Oltremare, Napoli, 2008.

${ }^{8}$ Per una ricostruzione delle vicende urbanistiche e architettoniche del Dodecaneso cfr. E. Papani Dean, La dominazione italiana e l'attività urbanistica ed edilizia nel Dodecaneso, 1912-1943, in «Storia Urbana», III, n. 8, maggio-agosto 1979, pp. 3-47; F.I. Apollonio, Architettura e città nel Dodecaneso, in Architettura italiana d'oltremare 1870-1940, a c. di G. Gresleri, P.G. Massaretti, S. Zagnoni, Venezia, Marsilio Editori, 1993, pp. 313-321; S. Martinoli, E. Perotti, Architettura coloniale italiana nel Dodecaneso. 1912-1943, Torino, Edizioni Fondazione Giovanni Agnelli, 1999.

${ }_{9}^{9}$ Paolo Nicoloso, Mussolini Architetto. Torino, Einaudi, 2008.
} 
continui interventi della dirigenza fascista mostrano uno spaccato significativo del modo di agire del regime, evidenziando la faciloneria e l'improvvisazione di una strategia politico-militare aggressiva, certamente influenzata, a partire dal 1938, dalla rapida evoluzione del contesto internazionale, ma senza dubbio basata su aspirazioni sproporzionate alle reali possibilità del paese.

\section{Conclusioni}

II filo rosso delle relazioni con l'Italia, una storia che parte da lontano, dall'antichità romana e si stringe nel corso dei secoli. Furono vastissimi i progetti che il governo italiano promosse su tutto il territorio albanese, durante il ventennio fascista. Sono più di 20.000 i disegni che restituiscono puntualmente le fasi del processo costruttivo delle "moderne" città albanesi: un programma ambizioso e lungimirante di pianificazione che ha prodotto una straordinaria quantità di elaborati. Essi testimoniano il ruolo della cultura italiana, la sua capacità di trasferire i modelli consolidati dell'Urbe Massima, di divulgarli e promuoverli fino a renderli fruibili e accessibili alle successive generazioni di governo.

A tali progetti parteciparono ingegneri e architetti ${ }^{10}$ come Armando Brasini, Florestano di Fausto, Gherardo Bosio, Vittorio Ballio Marpurgo, Ferdinando Poggi, Ivo Lambertini, di artisti come Antonio Maraini, Giuseppe Gronchi, Giulio Rosso, di paesaggisti come Pietro Porcinai e gli fratelli Sgaravatti.

Fin dal 1939, con l'insediamento dell'Ufficio Centrale a Tirana, sono varati i primi programmi di lavori, per il "miglioramento urbanistico ed edilizio" delle principali città albanesi. Essi intrecciano alcune coordinate che costituiscono una griglia costante di riferimento, riguardante: il rapporto con la città esistente, qualificazione del centro politico, amministrativo, religioso, creazione di un asse commerciale, zoning e decentramento, soluzione della viabilità interna ed esterna, potenziamento delle risorse ambientali. Questi temi saranno poi approfonditi per la stesura dei piani regolatori regionali redatti tra il 1942 e il 1943.

La scelta va dunque nella direzione di un professionismo di scuola romana, apprezzato proprio per la capacità di fondere "mirabilmente" gli elementi dell'architettura locale col linguaggio "austero" della romanità. Tirana, Durazzo e Valona, furono al centro delle prime trasformazioni urbane e infrastrutturali varate da società italiane. II processo protezionistico dell'Italia fascista sull'Albania ${ }^{11}$ si strinse attorno ad alcuni provvedimenti decisivi, come il proclama d'Agirocastro del 1917 e la Dichiarazione degli Ambasciatori del 1921. Sono gli anni in cui l'impegno italiano per l'indipendenza e la tutela del territorio albanese si rafforza col Patto d'Amicizia e sicurezza 1926, che individua l'Italia come partner preferenziale, ponendo le basi per programmare e gestire una struttura economica, ritenuta troppo debole per poter rispondere alle esigenze delle nuove istituzioni. II fascismo affronta fin dall'inizio i nodi fondamentali dello sviluppo, riguardanti i meccanismi d'investimento e la programmazione dei lavori pubblici, che devono rispondere anche a una strategia di trasferimento di ricchezze e di capitali. Prova ne è la costituzione, nel 1925 di una Società per lo Sviluppo Economico Albanese (S.V.E.A), promossa, insieme alla Banca Nazionale d'Albania, da un gruppo finanziario italiano, sulla base di precedenti atti riguardanti convenzioni per il prestito per i lavori pubblici.

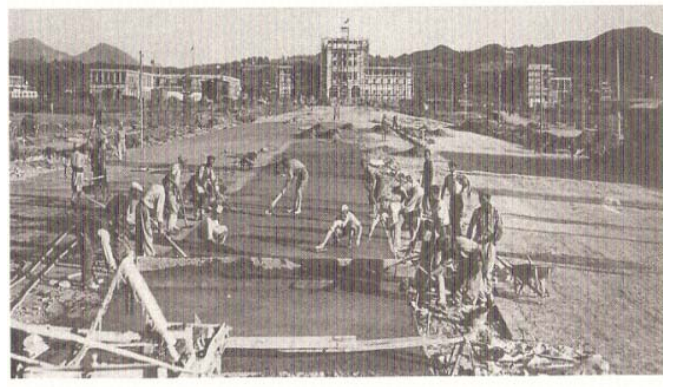

Fig. 3. La costruzione del Viale dell'Impero (Foto Luce, 20 dicembre 1939 Tirana).

\footnotetext{
10 Paolo Portoghesi, I grandi architetti del Novecento. Roma, Newton \& Compton Editori, 1998.

${ }^{11}$ Giuliano Gresleri, "1936-40: Programma e strategia delle «<citta' imperiali >>, in Architettura italiana d'oltremare: 1870-1940, eds. Giuliano Greseri, Pier Giorgio Massaretti, e Stefano Zagnoni (Venezia: Marsilio, 1993), 178-201: e Gresleri, "Architecture for the Empire", Rassegna, 14 (September 1992):36-51.
} 


\section{References}

Art \& Trashegimi, nr. 4, Dossier Tirana, Rikthimi i Muzave,maj 2012.

Emin Riza. Historia e arkitektures shqiptare nga fillimet deri 1912. Tirane, 1987.

Giuliano Gresleri, Massaretti, Pier Giorgio, Zagnoni, Stefano. Architettura italiana d'oltremare 1870-1940. (1993) Venezia: Marsilio,1993.

G. Gresleri, La via dell'est: da Tirana a Lubiana, in Architettura italiana d'oltremare 1870-1940, a c. di G. Gresleri, P.G. Massaretti, S. Zagnoni, Venezia, Marsilio Editori, 1993, pp. 323-331. Cfr. L. Billeri, Bosio e i piani urbanistici per le città dell'Albania, in Gherardo Bosio. Architetto fiorentino, 1903-1941.

ICCROM Working Group 'Heritage and Society'. Definition of Cultural Heritage, References to Documents in History. Selected by J. Jokilehto. (Originally for ICCROM, 1990). Revised for CIF: 15 January 2005.

L'amicizia tra Italia e Albania: passato, presente, futuro. (2006) La presenza italian in Albania nella prima meta' del XX secolo.Complesso di Vicolo Valdina, Roma 21-26 novembre 2006.

Maria Adriana Giusti. Albania, Architettura e Citta' 1925-1943. (2006) Finito di stampare nella tipografia Bandecchi \& Vivaldi, Pontedera per conto di artout - Maschietto Editore, Firenze Dicembre 2006.

Maria De Santis, Giuseppe Terragni. Casa del Fascio, Como, 1932-36, in Costruire in laterizio. Grandi Maestri. n. 117, maggio-giugno 2007.

Massimo Locci, Nuova Banca di Albania Memoria e Innovazione. Concorso per il restauro e l'ampliamento della banca central a Tirana.

Nerital Ceka. Apollonia: history and monuments. Tirana, 2005.

Paolo Portoghesi, I grandi architetti del Novecento. Roma, Newton \& Compton Editori, 1998.

Paolo Nicoloso, Mussolini Architetto. Torino, Einaudi, 2008.

K. Miho, I. Sukaj, P. Thomo, V. Bushati. Historia e arkitektures ne Shqiperi (1912-1944). (1988) Shtepia Botuese e Librit Universitar.Tirane, 1988.

Koco Miho, Profesor arkitekt Kristo Sotiri, Jeta dhe vepra. (2005) Botimi i dyte. Tirane, 2005.

Sculture di Antonio Maraini dal 1908 al 1942. (1944) Album realizzato dall'artista nel 1942; il memoriale dell'artista, radatto nel Settembre 1944.

The buildind of the bank of Albania. Brochure. Tirane 2003.

Vittorio Santoianni, II Razionalismo nelle colonie italiane 1928-1943. La «nuova architettura» delle Terre d'Oltremare, Napoli, 2008. 